\title{
Strong third-order Kerr nonlinearity in 2D PdSe 2 dichalcogenide films
}

\author{
Linnan Jia ${ }^{a}$, Jiayang $\mathrm{Wu}^{\mathrm{a}}$, Tieshan Yang ${ }^{\mathrm{b}}$, Baohua Jia ${ }^{\mathrm{a}, \mathrm{b}}$, David J. Moss ${ }^{\mathrm{a}, *}$ \\ ${ }^{a}$ Centre for Micro-Photonics, Swinburne University of Technology, Hawthorn, VIC 3122, Australia \\ ${ }^{b}$ Centre for Translational Atomaterials, Swinburne University of Technology, Hawthorn, VIC 3122, Australia \\ *E-mail:dmoss@swin.edu.au
}

Abstract: We characterize the third-order optical nonlinearity in $\mathrm{PdSe}_{2}$ dichalcogenide films via the Z-Scan technique. A strong and negative (self-defocusing) Kerr nonlinearity $\left(n_{2}\right)$ of $7.65 \times 10^{-16} \mathrm{~m}^{2} / \mathrm{W}$ is observed at $800 \mathrm{~nm}$.

\section{Introduction}

Transition metal dichalcogenides (TMDCs), such as $\mathrm{MoS}_{2}$ [1], $\mathrm{MoSe}_{2}[2]$, and $\mathrm{WS}_{2}$ [3] are promising nonlinear optical materials for future photonic devices. Palladium diselenide $\left(\mathrm{PdSe}_{2}\right)$, a new 2D noble metal dichalcogenide in the TMDC family, has recently become of significant interest. Similar to the puckered structure of black phosphorus (BP), $\mathrm{PdSe}_{2}$ has a puckered pentagonal atomic structure - with one Pd atom bonding to four Se atoms and two adjacent Se covalently bonding with each other [4]. This low-symmetry structure makes PdSe 2 possess unique in-plane anisotropic optical and electronic properties, featuring a noncentrosymmetric (pentagonal) structure, in contrast with its cousin $\mathrm{PtSe}_{2}[5,6]$. Further, the bandgap of $\mathrm{PdSe}_{2}$ is strongly layer-dependent, varying form $0 \mathrm{eV}$ (bulk) to $1.3 \mathrm{eV}$ (monolayer), a property well suited for photonic and optoelectronic applications [4, 7]. Most significantly, $\mathrm{PdSe}_{2}$ is highly air-stable, indicating its robustness and potential for practical applications, in contrast with BP. The high carrier mobility [4, 7] and anisotropic Raman spectroscopy [8] of 2D PdSe 2 layers have been investigated as well as their high performance as photodetectors from the visible to mid-infrared wavelengths [7]. To date, however, its optical Kerr nonlinearity has not been investigated.

In this paper, we report measurements of the third-order nonlinear optical response of 2D PdSe $\mathrm{Pilms}_{2}$ Experimental results using the Z-scan technique at $800 \mathrm{~nm}$ show that $\mathrm{PdSe}_{2}$ films exhibit a strong and negative (self-defocusing) Kerr nonlinearity $\left(n_{2}\right)$ of $\sim-7.65 \times 10^{-16} \mathrm{~m}^{2} / \mathrm{W}$, which is two orders of magnitude higher than that of bulk silicon. Our results show that the extraordinary third-order nonlinear optical properties of $\mathrm{PdSe}_{2}$ have a strong potential for high-performance nonlinear photonic devices.

\section{Material preparation and characterization}

Fig. 1(a) shows the atomic structure of $\mathrm{PdSe}_{2}$ crystals. Different from other TMDCs like $\mathrm{MoS}_{2}$ and $\mathrm{WS}_{2}, \mathrm{PdSe}_{2}$ has a unique puckered pentagonal structure, where the Se-Pd-Se layers stack with weak van der Waals interactions to form a layered structure [4, 7]. In each monolayer, the pentagonal rings are formed with one Pd atom bonding to four Se atoms and two adjacent Se covalently bonding with each other, which is similar to the puckered structure of $\mathrm{BP}$ and results in the low inversion symmetry properties of $\mathrm{PdSe}_{2}$.

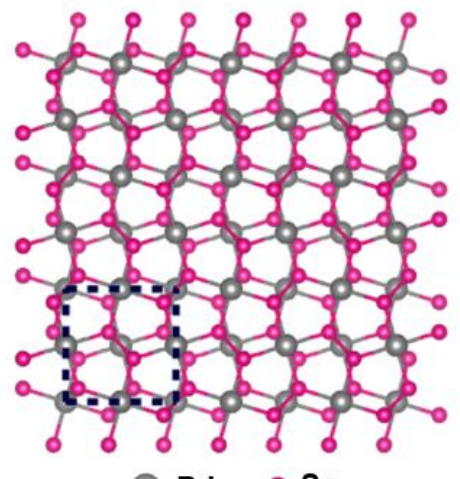

Pd $\mathrm{Pde}$

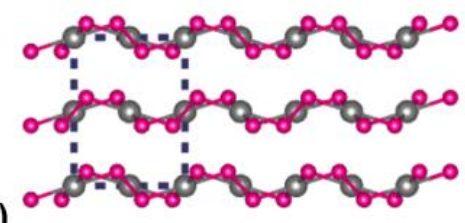

(a)

Fig. 1 (a) Schematic atomic structure of $\mathrm{PdSe}_{2}$, (b) AFM height profile and (c) Raman spectrum of multilayer PdSe $\mathrm{Pdilm}_{2}$.

Multilayer $\mathrm{PdSe}_{2}$ large-scale films used in our experiment were synthesized on the transparent sapphire substrate via the chemical vapor deposition (CVD) method. The morphology image and height profile of the $\mathrm{PdSe}_{2}$ film was characterized by the atomic force microscopy (AFM), as shown in Fig. 1(b). The measured thickness is $\sim 8$ nm. Fig. 1(c) shows the Raman spectrum of PdSe 2 film with an excitation laser wavelength at $514 \mathrm{~nm}$. Three 
typical phonon modes of $\mathrm{A}_{\mathrm{g}}{ }^{1}\left(\sim 145.5 \mathrm{~cm}^{-1}\right), \mathrm{B}_{1 \mathrm{~g}}{ }^{2}\left(\sim 222.5 \mathrm{~cm}^{-1}\right)$, and $\mathrm{A}_{\mathrm{g}}{ }^{3}\left(\sim 258.8 \mathrm{~cm}^{-1}\right)$ are observed, where the $\mathrm{A}_{\mathrm{g}}{ }^{1}$ and $\mathrm{B}_{1 \mathrm{~g}}{ }^{2}$ vibrational modes correspond to the movement of Se atoms and the $\mathrm{A}_{\mathrm{g}}{ }^{3}$ mode relates to the relative movements between Pd and Se atoms [7, 8].

\section{Z-scan measurements}

The third-order optical nonlinear response of the $\mathrm{PdSe}_{2}$ films was characterized via the open- (OA) and closedaperture (CA) Z-scan methods [9]. A femtosecond laser source at $800 \mathrm{~nm}$ wavelength was used to excite the samples, with a laser pulse duration of $\sim 140$ fs. Fig. 2(a) shows the OA Z-scan curve for the PdSe $\mathrm{P}_{2}$ film at the (incident) laser intensity of $17.15 \mathrm{GW} / \mathrm{cm}^{2}$. Typical reverse saturation absorption (RSA) can be observed, with the transmission decreasing as the $\mathrm{PdSe}_{2}$ sample was moved through the focal point. Various mechanisms, including two-photon absorption (TPA) and free carrier absorption (FCA), may contribute to the RSA behavior $[9,10]$. By fitting the OA data, the nonlinear absorption coefficient $\alpha_{\mathrm{NL}}$ is measured to be $\sim 2.82 \times 10^{-8} \mathrm{~m} / \mathrm{W}$, which is comparable to the values of graphene [11] and $\mathrm{WS}_{2}[3]$.
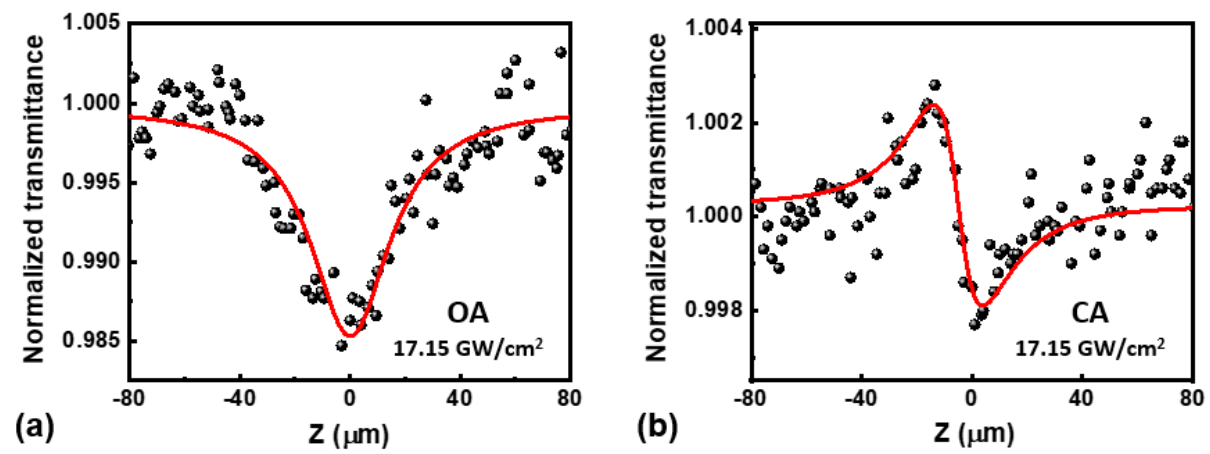

Fig. 2 Measured (a) OA and (b) CA curves of $\mathrm{PdSe}_{2}$ film at laser intensity of $17.15 \mathrm{GW} / \mathrm{cm}^{2}$.

We also characterize the Kerr nonlinearity of $\mathrm{PdSe}_{2}$ films via the CA Z-scan method. Fig. 2(b) displays the CA data of the multilayer $\mathrm{PdSe}_{2}$ film. Obviously, the normalized transmittance of sample exhibits a transition from peak to valley when the sample passes the focal plane. The typical peak-valley CA configuration reflects a negative Kerr coefficient $n_{2}$, indicating the optical self-defocusing effect in $8 \mathrm{~nm}-\mathrm{PdSe} \mathrm{e}_{2}$ film. The measured $n_{2}$ of $\mathrm{PdSe}_{2}$ is $\sim-7.65 \times 10^{-16} \mathrm{~m}^{2} / \mathrm{W}$ at an intensity of $17.15 \mathrm{GW} / \mathrm{cm}^{2}$. Compared with other 2D materials (Table 1), $n_{2}$ for $\mathrm{PdSe}_{2}$ is lower than graphene and BP, but still two orders of magnitude higher than bulk silicon [12-14], which demonstrates the high potential of $\mathrm{PdSe}_{2}$ as a new optical material for nonlinear photonic applications. Further, like Si-Ge heterostructures, [15] $\mathrm{PdSe}_{2}$ may also offer interesting possibilities for second order nonlinear effects courtesy of its complex anisotropic nonlinear optical characteristics.

Table 1. Comparison of $\alpha_{\mathrm{NL}}$ and $n_{2}$ of various 2D layered materials

\begin{tabular}{cccccc}
\hline Material & Laser parameter & Thickness & $\alpha_{\mathrm{NL}}(\mathrm{m} / \mathrm{W})$ & $n_{2}\left(\mathrm{~m}^{2} / \mathrm{W}\right)$ & Reference \\
\hline Graphene & $1150 \mathrm{~nm}, 100 \mathrm{fs}$ & $5-7$ layers & $3.8 \times 10^{-8}$ & $-5.5 \times 10^{-14}$ & {$[11]$} \\
$\mathrm{MoS}_{2}$ & $1064 \mathrm{~nm}, 25 \mathrm{ps}$ & $25 \mu \mathrm{m}$ & $(-3.8 \pm 0.59) \times 10^{-11}$ & $(1.88 \pm 0.48) \times 10^{-16}$ & {$[1]$} \\
$\mathrm{WS}_{2}$ & $1040 \mathrm{~nm}, 340 \mathrm{fs}$ & $57.9 \mathrm{~nm}$ & $(1.81 \pm 0.08) \times 10^{-8}$ & $(-3.36 \pm 0.27) \times 10^{-16}$ & {$[3]$} \\
$\mathrm{PtSe}_{2}$ & $800 \mathrm{~nm}, 150 \mathrm{fs}$ & $4.6 \mathrm{~nm}$ & $-8.80 \times 10^{-8}$ & - & {$[5]$} \\
$\mathrm{PtSe}_{2}$ & $1030 \mathrm{~nm}, 340 \mathrm{fs}$ & 17 layers & - & $(-3.76 \pm 0.46) \times 10^{-15}$ & {$[6]$} \\
$\mathrm{PdSe}_{2}$ & $800 \mathrm{~nm}, 140 \mathrm{fs}$ & $8 \mathrm{~nm}$ & $(2.82 \pm 0.16) \times 10^{-8}$ & $(-7.65 \pm 0.83) \times 10^{-16}$ & This work \\
\hline
\end{tabular}

\section{References}

[1] S. Bikorimana et al., "Nonlinear optical responses in two-dimensional transition metal dichalcogenide multilayer: $\mathrm{WS}_{2}, \mathrm{WSe}_{2}, \mathrm{MoS}_{2}$ and $\mathrm{Mo}_{0.5} \mathrm{~W}_{0.5} \mathrm{~S}_{2}$," Opt. Exp. 24(18), 20685-95 (2016).

[2] G. Wang et al., "Broadband saturable absorption and exciton-exciton annihilation in MoSe 2 composite thin films," Opt. Mater. Exp. 9(2), 483-496 (2019).

[3] N. Dong et al., "Dispersion of nonlinear refractive index in layered $\mathrm{WS}_{2}$ and $\mathrm{WSe}_{2}$ semiconductor films induced by two-photon absorption," Opt. Lett. 41(17), 3936-3939 (2016).

[4] A. D. Oyedele et al., "PdSe 2 : Pentagonal 2D layers with high air stability for electronics," J. Am. Chem. Soc. 139(40), 14090-14097 (2017).

[5] G. Wang et al., "Ultrafast carrier dynamics and bandgap renormalization in layered PtSe 2 ," Small. 15(34), 1902728 (2019).

[6] L. Wang et al., "Nonlinear optical signatures of semiconductor to semimetal transition in PtSe ${ }_{2}, "$ Laser Photon. Rev. 13(9), 1900052 (2019).

[7] Q. Liang et al., "High-performance, room temperature, ultra-broadband photodetectors based on air-stable PdSe ${ }_{2}$," Adv. Mater. 31(24), 1807609 (2019).

[8] Jiang et al., "Anisotropic growth and STM of ultrathin even-layered PdSe 2 ribbons," Small, 15(45), 1902678 (2019).

[9] L. Jia et.al., "Highly nonlinear BiOBr nanoflakes for hybrid integrated photonics." APL Photon. 4(9), 090802 (2019).

[10] P. Olszak et.al., "Spectral and temperature dependence of TPA and free-carrier absorption in InSb." Phys. Rev. B 82(23), 235207 (2010).

[11] G. Demetriou et.al., "Nonlinear optical properties of multilayer graphene in the infrared," Opt. Exp. 24(12), 13033-13043 (2016).

[12] D. J. Moss et.al., "New CMOS-compatible platforms based on SiN and Hydex for nonlinear optics," Nat. Photon. 7(8), 597-607 (2013).

[13] Y. Yang et.al., "Enhanced FWM in waveguides integrated with graphene oxide." APL Photon. 3(12), 120803 (2018).

[14]J.Wu et.al., "Enhanced nonlinear FWM in MRRs integrated with layered GO films", in press, Small (2020). DOI: 10.1002/smll.201906563

[15] E. Ghahramani, D.J. Moss, and J.E. Sipe, "Large $2^{\text {nd }}$ order nonlinear optical response of strained odd period Si/Ge superlattices on (001) Si substrates", Physical Review Letters vol. 64, no. 23, pp.2815-2818 (1990). DOI:10.1103/PhysRevLett.64.2815. 